\title{
Single-docking robotic-assisted artery-guided segmental splenic flexure colectomy for splenic flexure cancer -a propensity score-matching analysis
}

\author{
Tao Zhang ${ }^{1 \#}$, Zijia Song ${ }^{1 \#}$, Yaqi Zhang', Xiaopin Ji ${ }^{1}$, Xiaoqian Jing ${ }^{1}$, Yi Shi' ${ }^{2}$ Xi Cheng ${ }^{1}$, Ren Zhao ${ }^{1}$ \\ ${ }^{1}$ Department of General Surgery, Ruijin Hospital, Shanghai Jiaotong University School of Medicine, Shanghai, China; ${ }^{2}$ Department of General \\ Surgery, Shanghai Ninth People's Hospital, Shanghai Jiaotong University School of Medicine, Shanghai, China \\ Contributions: (I) Conception and design: R Zhao, T Zhang; (II) Administrative support: X Cheng, X Ji; (III) Provision of study materials or patients: \\ T Zhang, i Shi; (IV) Collection and assembly of data: T Zhang X Jing, Y Shi; (V) Data analysis and interpretation: Z Song, Y Zhang; (VI) Manuscript \\ writing: All authors; (VII) Final approval of manuscript: All authors. \\ \#These authors contributed equally to this work. \\ Correspondence to: Ren Zhao, MD, PHD, FACS; Xi Cheng, MD, PhD. Department of General Surgery, Ruijin Hospital, Shanghai Jiaotong \\ University School of Medicine, Shanghai, China. Email: rjzhaoren@139.com; drchengxi@126.com.
}

Background: Splenic flexure cancer (SFC) is a rare condition in colorectal cancer (CRC). The appropriate surgical treatment for SFC remains controversial. In recent years, we have used artery-guided segmental splenic flexure colectomy (ASFC) to treat SFC in which robotic access is gradually applied. The study sought to assess the clinical and oncologic outcomes of robotic-assisted ASFC compared to laparoscopic-assisted ASFC for SFC by undertaking a propensity score-matching analysis.

Methods: Seventy patients underwent a robotic-assisted ASFC $(n=19)$ or laparoscopic-assisted ASFC $(n=51)$ to treat SFC from Dec 2015 to Dec 2019. Their data were prospectively collected. The patients were matched at a ratio of 1:1 according to sex, age, body mass index (BMI), comorbidities, the American Society of Anesthesiologists (ASA) score ( $\leq 2$ or $>2$ ), previous abdominal surgeries, and pathologic stage.

Results: No statistically significant differences were found between the robotic- and laparoscopic-assisted ASFC groups in relation to operation time, estimated blood loss, length of postoperative hospital stay, time to liquid diet, postoperative complications, tumor size, distal resection margins, histology, lymph node harvest, metastatic lymph nodes, and neuro-vascular invasion. Additionally, no case was converted to a laparotomy. There were no cases readmission or mortality within 30 days of surgery. The distal resection margins were longer in the robotic-assisted ASFC group than the laparoscopic-assisted ASFC group. The robotic-assisted ASFC group had significantly higher operation expenses than the laparoscopic-assisted ASFC group. However, there was no significant difference in the surgical material expenses between the two groups. There were 2 cases of complications in each group; both cases were classified as grade I or II under Dindo's classification of surgical complications.

Conclusions: With the exception of operation expenses, robotic-assisted ASFC rivals laparoscopic-assisted ASFC in many respects. ASFC meets the recommended oncological criteria in terms of resection margins and lymph node harvest. We await the results for the long-term oncologic outcomes.

Keywords: Splenic flexure cancer (SFC); robotic-assisted surgery; laparoscopic surgery; artery-guided segmental splenic flexure colectomy

Submitted Apr 02, 2021. Accepted for publication May 27, 2021.

doi: 10.21037/jgo-21-221

View this article at: http://dx.doi.org/10.21037/jgo-21-221 


\section{Introduction}

Colorectal cancer (CRC) is the $3 \mathrm{rd}$ most common malignancy worldwide (1). In 2018, there were over 1 million new cases of CRC and more than 550,000 CRCrelated deaths [2018] (1). Splenic flexure cancer (SFC), which is among the different colonic locations for CRC, accounts for approximately $2-8 \%$ of all CRCs (2-4). Unlike sigmoid cancer, which represents more than $80 \%$ of leftsided cancer, SFC is a rare condition that is associated with poor prognosis and a high risk of colonic obstruction (4-6).

After almost two decades of development, robotic-assisted surgery is no longer a novelty. Due to its stability, dexterity, control, three-dimensional (3D) high-definition display, and subsequent accuracy and precision in anatomical dissection, it is increasingly being applied to colorectal surgery.

Due to the limited range of motion of the robotic arms of the Si system, reports of robotic left colectomies are rare. Korean surgeon Bae (7) reported using a dualdocking technique to maximize splenic flexure mobilization in robotic-assisted LHCs (7). Upper docking was used to dissect the transverse colon and splenic flexure, and lower docking was used to dissect the inferior mesenteric artery (IMA) and sacral promontory. Today, with the recognition of the importance of $\mathrm{CME}$, and the guarantee of adequate resection margin and harvested lymph nodes (D2 or D3) in colonic cancer, more surgeons prefer the segmental splenic flexure colectomy to the extended right colectomy (ERC) or the left hemicolectomy (LHC) in treating SFC.

We have been performing robotic-assisted colorectal surgery with a Si system since 2015 . We have searched for a technique of single-docking robotic-assisted artery-guided segmental splenic flexure colectomy (ASFC) for SFC with a dissection range from the left branch of the mid-colic artery to the sigmoid artery, which differed from other present concept of the range of resection. This study sought to assess the clinical and oncologic outcomes of roboticassisted ASFC compared to laparoscopic-assisted ASFC for SFC. It should be noted that the long-term oncologic outcomes will be examined once the long-term survival data have been collected. We present the following article in accordance with the STROBE reporting checklist (available at http://dx.doi.org/10.21037/jgo-21-221).

\section{Methods}

\section{Patients and data collection}

Seventy-three patients underwent laparoscopic- or robotic- assisted ASFCs to treat SFC at Ruijin Hospital, Shanghai Jiaotong University School of Medicine from December 2015 to December 2019. All of the operations were carried out by the same qualified and well-experienced surgical team, who had performed more than 1,000 laparoscopicassisted colectomies. Patients' information was prospectively collected and retrospectively analyzed. Fifty one patients who underwent laparoscopic-assisted surgery and 19 patients who underwent robotic-assisted surgery were included in this study. Three patients with metastasis were excluded from the study.

The study was approved by the Institutional Review Board of Ruijin Hospital, Shanghai Jiaotong University School of Medicine, and informed consent forms were obtained from all patients for the operations. The study was performed in compliance with the Declaration of Helsinki (as revised in 2013).

Patients' medical records were reviewed, and the following data were collected: sex, age, body mass index (BMI), comorbidities, the American Society of Anesthesiologists (ASA) score $(\leq 2$ or $>2)$, previous abdominal surgeries, pathologic stage, operation time, estimated blood loss, time to liquid diet, length of postoperative hospital stay (PHS, intraoperative morbidity, conversion to laparotomy, readmission and mortality within 30 days of surgery, postoperative complications, tumor size, histology, proximal and distal resection margins, lymph node harvest, metastatic lymph nodes, neuro-vascular invasion, and pathologic stage data. Pathologic stage was classified according to the 8th Edition of the AJCC Cancer Staging Manual, and postoperative complications were graded according to the Clavien-Dindo classification system.

\section{Surgical procedure}

\section{Bowel preparation}

The patients were asked to go on a liquid diet 1-2 days before the operation. Two liters of polyethylene glycol electrolyte solution was used as a preoperative colonic lavage. Prophylactic antibiotics were given to all the patients.

\section{Installation and docking}

The patients were kept in the Trendelenburg position at $30^{\circ}$, tilted left-side up at an angle of $30^{\circ}-45^{\circ}$ during the operation with general anesthesia. 5 ports (i.e., a $12-\mathrm{mm}$ camera port, a $12-\mathrm{mm}$ port for the assistant, and three 8-mm robotic working ports $\mathrm{R} 1-3)$ were applied. To 


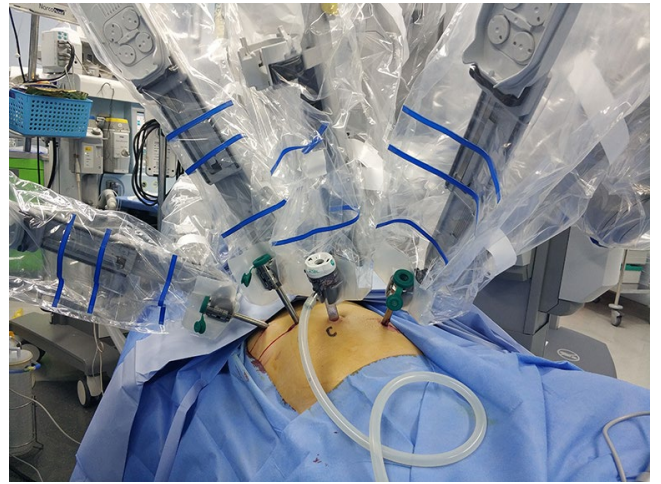

Figure 1 Docking.

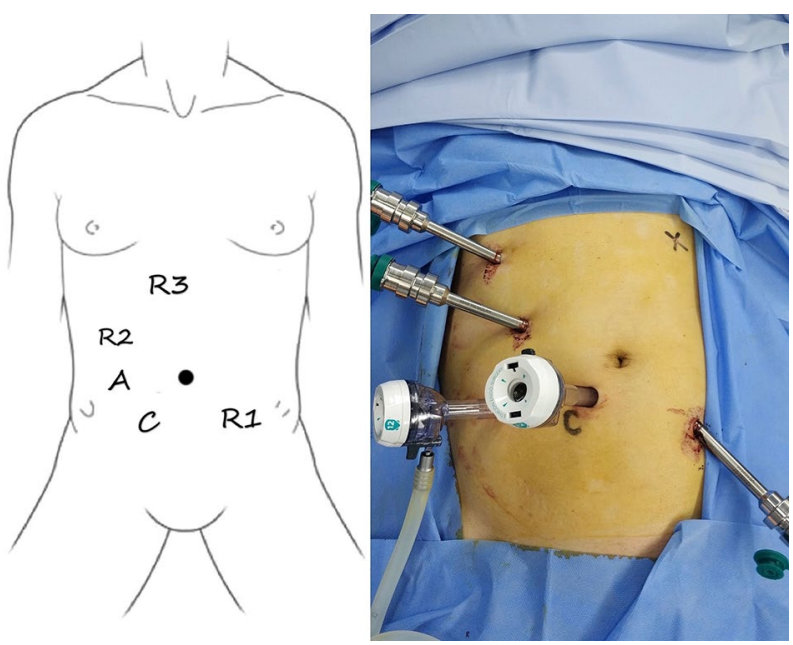

Figure 2 Port sites.

insert the robotic scope, the $12 \mathrm{~mm}$ trocar was placed $2-3 \mathrm{~cm}$ to the lower right of the umbilicus. R1 was placed approximately $8 \mathrm{~cm}$ to the lower left of the umbilicus. R2 was inserted at the right mid clavicular line, $3-5 \mathrm{~cm}$ below the right costal margin. R3 was inserted $2-3 \mathrm{~cm}$ left of and below the xyphoid process. The assistant trocar was placed in the middle of the scope trocar and R2. The surgical cart was placed on the left side of the head, oblique to the surgical table. R1 was armed with the ultrasonographic scalpel, corresponding to the right hand, R3 with the double-fenestrated forceps, and R2 with the fenestrated bipolar forceps, both corresponding to the left hand (see Figures 1,2).

\section{Bowel mobilization and lymphovascular dissection}

The surgical procedure followed the principle of complete mesocolectomy, and the dissection was initiated from the origin of the inferior mesenteric vein (IMV). With the exposure of the Treitz ligament, the IMV could be easily dissected and ligated. As the Toldt's space was then traced and explored further, the ureter and gonadal vein were finely preserved, and the Gerota's fascia was left completely untouched. After the dissection of No. $253 \mathrm{LN}$, the root of the IMA was carefully dissected to protect the superior hypogastric plexus around the aortic bifurcation, and the locations of the gonadal vessels and ureter were reassessed. The left colic artery (if there was an ascending one) and the sigmoid artery were ligated with clips. The sigmoid colon and the lower part of the descending colon were dissected from the lateral. We then came to the splenic flexure, which, in a survey of experienced laparoscopic colorectal surgeons, was cited as the most difficult among the laparoscopicassisted colorectal procedures (5). As the IMV was already lifted, the root of the transverse colon was able to be freely dissected from the lower border of the pancreas. The exposure of the retroperitoneal space was accomplished by lifting the mesocolon with the 3 rd-arm, which subsequently facilitated surgical access and visualization. As all the medial procedures were completed, we started laterally from the gastrocolic ligament. The $3 \mathrm{rd}$ arm lifted the stomach up, and the ligament was opened outside the epigastric arc to preserve the blood supply of the stomach. Next, we were able to easily access the lesser sac, as the lower border of the pancreas was already freed, and we cut the mesocolon along the pancreas to the tail. At this point, great attention must be paid to protect the structure of the hilus lienis. The $3^{\text {rd }}$ arm was used to retract the descending colon to the medial side, the left paracolic gutter was dissected, meeting the previous plane of the left Toldt's fascia and the mobilized sigmoid colon.

\section{Anastomosis}

The specimen was extracted extracorporeally through a small epigastric midline incision, and a side-to-side anastomosis was accomplished using a double-stapling technique (see Figure 3). A 24-Fr catheter was retained in the left parietocolic gutter.

\section{Laparoscopic-assisted procedure}

The laparoscopic-assisted procedure adopted in this study was quite similar to the robotic-assisted procedure and has been well-documented elsewhere; thus, it has not been outlined again in this article. 


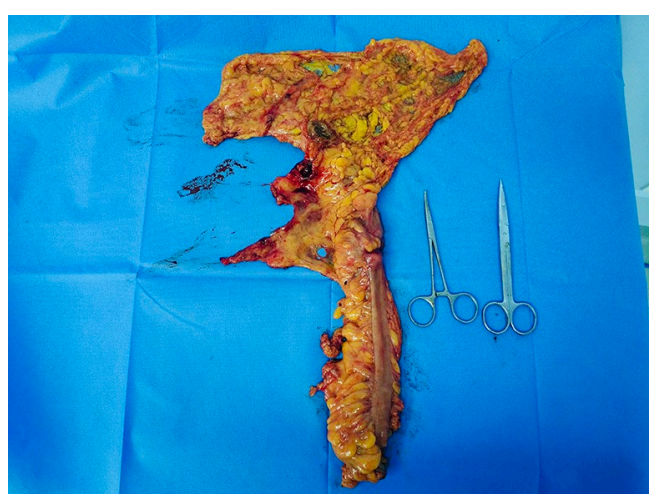

Figure 3 Specimen.

\section{Matching}

To reduce the bias of this retrospective, non-randomized control study, 1:1 propensity score-matching analysis using a bivariate logistic regression was conducted. The covariates were sex, age, BMI, previous abdominal surgery, ASA grade, comorbidities, and pathologic stage.

\section{Statistical analysis}

SPSS (version 22.0, SPSS Inc. Chicago, IL, USA) software was used for the statistical analysis. The Student's $t$ test, Mann-Whitney $U$ test, $\chi^{2}$ test, and Fisher exact test were used to evaluate whether the differences were statistically significant as appropriate. A P value $<0.05$ was considered statistically significant.

\section{Results}

\section{Baseline characteristics}

There was no significant difference between the two groups before matching; however, the matching still improved the consistency of the two groups in relation to the male-tofemale ratio, comorbidities, and pathological staging (see Table 1).

\section{Intraoperative and perioperative outcomes}

There was no significant statistical difference between the robotic- and laparoscopic-assisted ASFC groups in terms of operation time, estimated blood loss, length of PHS, time to liquid diet, and postoperative complications. Additionally, no case was converted to a laparotomy. There were no cases of readmission or mortality within 30 days of surgery. The operative duration ran from the beginning of the anesthesia to the end of all surgical procedures. As we started draping and docking when the anesthesiologist started infusion, basically no extra time was consumed. There were 2 cases of complications in each group. Specifically, in the roboticassisted ASFC group there was 1 case of anastomotic leakage and 1 case of pulmonary infection. In the laparoscopicassisted group, there was 1 case of gastroparesis and 1 case of transient ischemic attack (TIA). According to Dindo's classification of surgical complications, all cases were grade I or II. All of the above complications were treated using non-operative interventions. The anastomotic leakage was treated by a sustained para-anastomotic drainage and a douche, and the patient was discharged on the $19^{\text {th }}$ day after surgery on a normal diet. The pulmonary infection was treated with sensitive antibiotics. The patient with gastroparesis recovered with sustained gastric decompression, and was placed back on a liquid diet 7 days afterwards. The patient with TIA self-recovered with some neurotrophic treatment and showed no aftereffects. There was 1 case of splenic injury during a laparoscopic-assisted surgery due to the complicated adhesion of the mesocolon and splenic capsule. The spleen was preserved and the ripped capsule was cauterized.

The operation expenses of the robotic-assisted group were significantly higher than that of the laparoscopicassisted group (7,975 vs. 6,345 RMB or 1,126 vs. 896 US dollars). Conversely, the surgical material expenses did not differ significantly between the two groups (see Table 2). The robotic-assisted surgery had an extra expense related to the machine-running fee in our country, which amounted to approximately 30,000 RMB (4,200 US dollars). Some of the patients had private insurance that largely covered their total expenses.

\section{Patbologic and oncologic outcomes}

There was no difference between the two groups in terms of tumor size, distal resection margins, lymph node harvest, histology, metastatic lymph nodes, and neuro-vascular invasion (see Table 3). The distal resection margins were longer in patients in the robotic-assisted group than those in the laparoscopic-assisted group. Due to postoperative pathological classifications of stage III or high-risk II, 10 patients who underwent laparoscopic-assisted surgery and 9 patients who underwent robotic-assisted surgery received adjuvant chemotherapy afterwards. As some of the cases were performed in the last 2 years, the 3 -year 
Table 1 Baseline characteristics

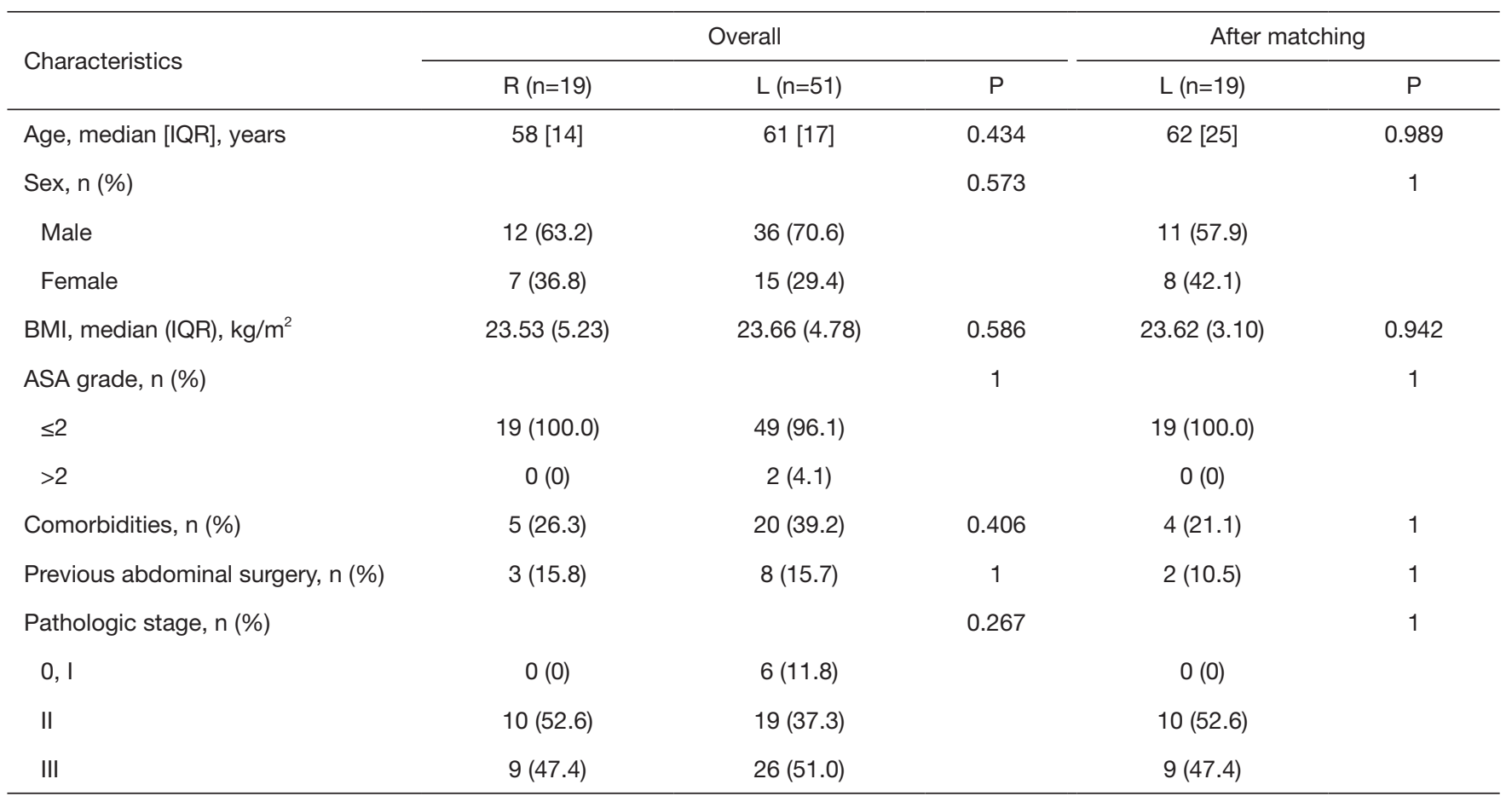

IQR, interquartile range; BMI, body mass index; ASA, The American Society of Anesthesiologists.

surveillance outcomes are not yet available.

\section{Discussion}

Controversy remains as to the appropriate surgical option for SFC. This controversy mainly relates to the extent of the colon resection, and thus the extent of the lymph node dissection. From an embryological point of view, the splenic flexure is an anatomical juncture at the end of the midgut and the beginning of hindgut. The three main options for treating SFC are segmental splenic flexure resection, left hemicolectomy (LHC), and extended right colectomy (ERC) (8).

In 2017, a nation-wide questionnaire was administered in France (9) in relation to surgical procedures for SFC to members of the French Association of Surgery, the French Federation of Surgical Research, the French Society of Digestive Surgery, and the French Research Group of Rectal Cancer Surgery. There was a strong consensus that patients with splenic flexure tumors should be treated with segmental splenic flexure resections and some limited consensus that they should be treated with left colic artery lymph node dissection (9). More than $70 \%$ of surgeons were of the view that splenic flexure resection was adequate in relation to the oncological quality of resection. The
5-7 cm margins of both ends and the harvesting of at least 12 lymph nodes guarantee the oncological safety of the removal of the primary tumor (9). The trend in France is also popular in other parts of the world.

Some retrospective research has been conducted to compare the segmental splenic flexure resection, the LHC, and the ERC to the lymphadenectomy. Ardu (10) reported that the segmental colectomy may not fulfill the recommended criteria that at least 12 lymph nodes be harvested, but found that the segmental colectomy was not more inferior in terms of OS and DFS than the ERC or LHC. Additionally, Faucheron et al. (11) reported that the segmental left colectomy had a relatively low morbidity and good function in SFC. Further, the survival rates were relatively low when comparing the literature.

As Fukuoka et al. noted (12), vascularization of the splenic flexure by the left colic artery occurred in only $40 \%$ of cases. In $21 \%$ of cases, the splenic flexure was vascularized by the middle colic artery only, and in $14 \%$ of cases, it was vascularized by both the left colic artery and the middle colic artery (12). Thus, the extent of surgical resection should include the origin ligation of the dominating artery, preserve the adjacent artery, and the en bloc lymphadenectomy. 
Table 2 Intraoperative and perioperative outcomes

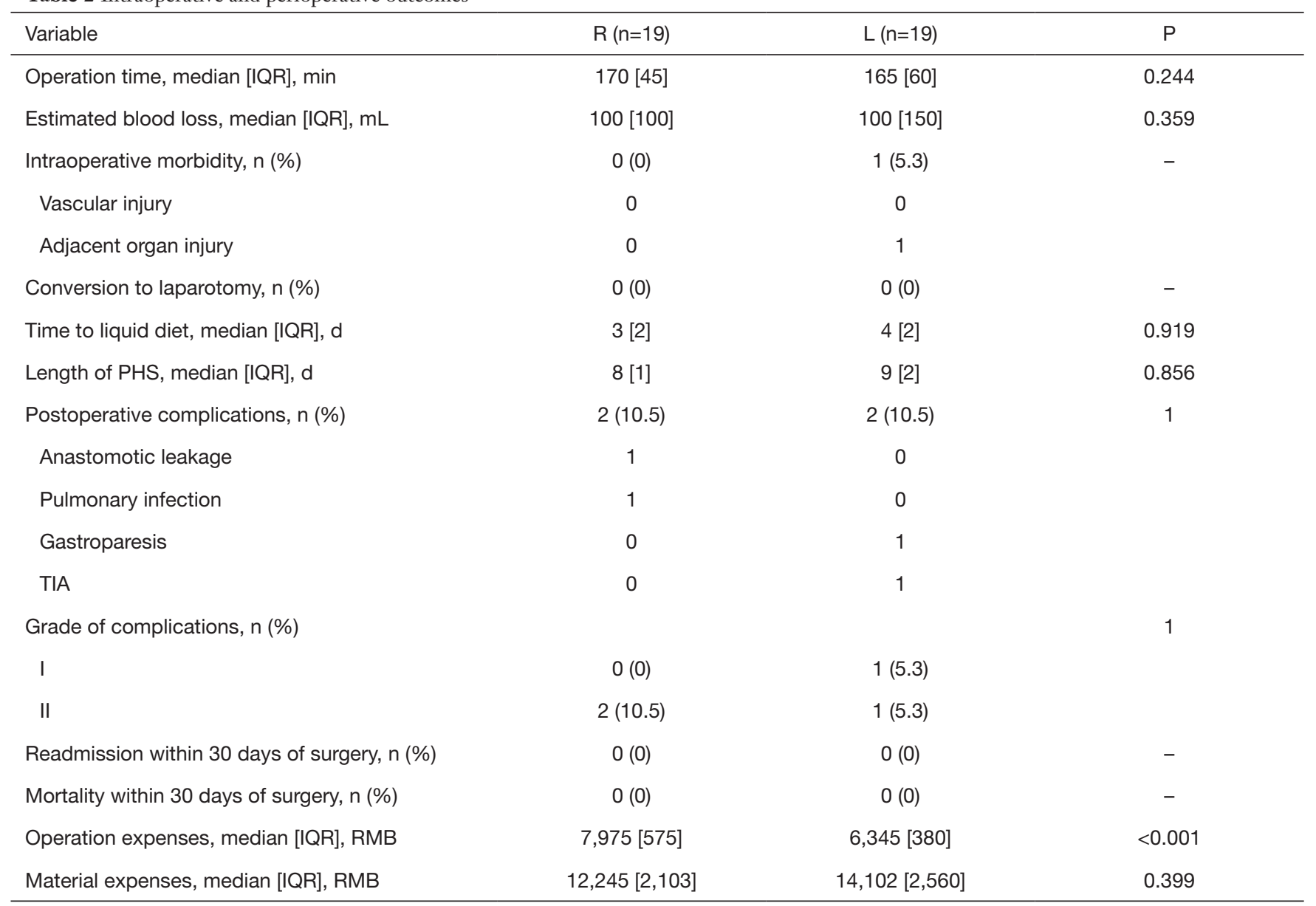

IQR, interquartile range; PHS, postoperative hospital stay; TIA, transient ischemic attack.

Table 3 Pathologic and oncologic outcomes

\begin{tabular}{lccc}
\hline Variable & $\mathrm{R}(\mathrm{n}=19)$ & $\mathrm{L}(\mathrm{n}=19)$ & $\mathrm{P}$ \\
\hline Tumor size, median [IQR], cm & $5[2]$ & $4[2]$ & 0.779 \\
Proximal resection margins, median [IQR], cm & $8[3]$ & $8[4]$ & 9.828 \\
Distal resection margins, median [IQR], cm & $10[5]$ & $15[8]$ & 0.034 \\
Lymph node harvest, median [IQR], $\mathrm{n}$ & $18[6]$ & & 0.702 \\
Histology, $\mathrm{n}(\%)$ & $14(73.7)$ & $11(57.9)$ \\
WD/MD & $5(26.3)$ & $8(42.1)$ & 0.495 \\
PD/others & $9(47.4)$ & $9(47.4)$ & 1 \\
Metastatic lymph node, $\mathrm{n}(\%)$ & $2(10.5)$ & $3(15.8)$ & 1 \\
Vascular invasion, $\mathrm{n}(\%)$ & $3(15.8)$ & $2(10.5)$ & 1 \\
Perineural invasion, $\mathrm{n}(\%)$ & & \\
\hline
\end{tabular}

$\mathrm{IQR}$, interquartile range; $\mathrm{WD}$, well differentiated; $\mathrm{MD}$, moderately differentiated; $\mathrm{PD}$, poorly differentiated. 
In the present study, the dissection range was from the left branch of the mid-colic artery to the sigmoid artery, which was less than that of a conventional LHC but much more than that of a segmental splenic flexure colectomy (SSFC). This is also why we named it the ASFC. The length of the distal and proximal margins in both groups were much longer than those reported for the SSFC in the literature for which $5-7 \mathrm{~cm}$ is common $(13,14)$. The distal margin was even longer in the robotic-assisted ASFC surgery. The according harvested lymph nodes were both more than the recommended number of $12(13,14)$.

In conclusion, robotic-assisted ASFC was not superior to laparoscopic-assisted ASFC in terms of operative duration, estimated blood loss, length of PHS, time to liquid diet, postoperative complications, material expenses, tumor size, distal margins, number of lymph nodes harvested, histology, metastatic lymph nodes, and neurovascular invasion. Robotic-assisted ASFC also results in a longer distal margin, which might be due to the precise anatomy of the left branch of the mid-colic vessels. As mentioned above, Korean surgeon Bae (7) reported that the dual-docking technique maximizes splenic flexure mobilization and has a median operation time of $227 \mathrm{~min}$ (with a range of 137 to $653 \mathrm{~min}$ ). In the present study, the median operation time for the robotic-assisted surgery was $170 \mathrm{~min}$, which represents a considerable time saving compared to the dual-docking method. Conversely, the robotic-assisted surgery cost more than the laparoscopic-assisted surgery. The operation expenses were approximately 1,600 RMB (225 USD) higher in the robotic-assisted group than the laparoscopic-assisted group; however, there was no difference between the two groups in terms of the material expenses, which was approximately 12,245 RMB (USD) in the robotic-assisted group and 14,102 RMB (1,980 USD) in the laparoscopicassisted group. Notably, the robotic-assisted surgery required an extra 30,000 RMB (4,200 US dollars) for the machine-running fee in our country, which was verified and established by the National Health Commission of PRC. Kim previously noted (15) that total hospital charges were approximately $40 \%$ greater for a robotic-assisted left colectomy with complete mesocolectomy (LCCM) than a laparoscopic-assisted LCCM (US\$14,800 vs. US\$10,500; $\mathrm{P}<0.001)$. Patients' private insurance mostly covered the total expenses of the robotic-assisted surgery (15). The situation is similar in our country.

The robotic-assisted ASFC did not present any advantage over the laparoscopic-assisted ASFC; however, it did provide surgeons with a better ergonomic experience (16). Catanzarite et al. (17) reviewed the ergonomics of surgery, and found that work-related musculoskeletal disorders are common among surgeons, with rates of $66-94 \%$ for open surgery, $73-100 \%$ for conventional laparoscopy, $54-87 \%$ for vaginal surgery, and $23-80 \%$ for robotic-assisted surgery (17). Long-shafted instruments, poor instrument handle design, and table and monitor positions represent unique risks in laparoscopic-assisted surgery. Roboticassisted surgery has some advantages; however, it is still associated with trunk, wrist, and finger strain. Additionally, first assistants of robotic-assisted surgery experience nonergonomic trunk, neck and shoulder angles. These findings are supported by a posture analysis. Tissue traction is reported as the most intensive action by the nurses (18). Ergonomic data was not collected in the present study. As the whole surgical procedure is single docking, there is no frequent change in the patient's position; thus, lowering the number of instrument replacements could lead to better ergonomic postures for first assistants.

\section{Conclusions}

Robotic-assisted ASFC rivals laparoscopic-assisted ASFC in many respects, but its main disadvantage is its high cost. In the near future, it is expected that robotic-assisted surgical systems will become more common, and the price of robotic-assisted procedures should approach those of laparoscopic-assisted procedures. Notably, the ASFC meets the recommended oncological criteria in terms of resection margins and lymph node harvest. We await the results for the long-term oncologic outcomes.

\section{Acknowledgments}

Funding: The study was supported by the National Natural Science Foundation of China (82002475 (X.C)), the Shanghai Sailing Program [20YF1427700 (XC)], the Shanghai Hospital Development Center [16CR2064B (RZ)], the Shanghai Municipal Health Construction Commission [201540026 (RZ)], the Youth Development Program of Ruijin Hospital, the Shanghai Jiaotong University School of Medicine, [2019QNPY01010 (TZ)], and the Shanghai Charity Cancer Foundation (TZ).

\section{Footnote}

Reporting Checklist: The authors have completed the 
STROBE reporting checklist. Available at http://dx.doi. org/10.21037/jgo-21-221

Data Sharing Statement: Available at http://dx.doi. org/10.21037/jgo-21-221

Conflicts of Interest: All authors have completed the ICMJE uniform disclosure form (available at http:// dx.doi.org/10.21037/jgo-21-221). Dr. TZ reports funding from Youth Development Program of Ruijin Hospital, the Shanghai Jiaotong University School of Medicine [2019QNPY01010 (TZ)], and the Shanghai Charity Cancer Foundation (TZ). Dr. XC reports from National Natural Science Foundation of China [82002475 (XC)], and the Shanghai Sailing Program [20YF1427700 (XC)]. Dr. RZ reports funding from Shanghai Hospital Development Center [16CR2064B (RZ)] and Shanghai Municipal Health Construction Commission [201540026 (RZ)]. The other authors have no conflicts of interest to declare.

Ethical Statement: The authors are accountable for all aspects of the work in ensuring that questions related to the accuracy or integrity of any part of the work are appropriately investigated and resolved. The study was approved by the Institutional Review Board of Ruijin Hospital, Shanghai Jiaotong University School of Medicine, and informed consent forms were obtained from all patients for the operations. The study was performed in compliance with the Declaration of Helsinki (as revised in 2013).

Open Access Statement: This is an Open Access article distributed in accordance with the Creative Commons Attribution-NonCommercial-NoDerivs 4.0 International License (CC BY-NC-ND 4.0), which permits the noncommercial replication and distribution of the article with the strict proviso that no changes or edits are made and the original work is properly cited (including links to both the formal publication through the relevant DOI and the license). See: https://creativecommons.org/licenses/by-nc-nd/4.0/.

\section{References}

1. Bray F, Ferlay J, Soerjomataram I, et al. Global cancer statistics (2018): GLOBOCAN estimates of incidence and mortality worldwide for 36 cancers in 185 countries. CA Cancer J Clin 2018;68:394-424.

2. Steffen C, Bokey EL, Chapuis PH. Carcinoma of the splenic flexure. Dis Colon Rectum 1987;30:872-4.
3. Aldridge MC, Phillips RK, Hittinger R, et al. Influence of tumour site on presentation, management and subsequent outcome in large bowel cancer. Br J Surg 1986;73:663-70.

4. Kim CW, Shin US, Yu CS, et al. Clinicopathologic characteristics, surgical treatment and outcomes for splenic flexure colon cancer. Cancer Res Treat 2010;42:69-76.

5. Levien DH, Gibbons S, Begos D, et al. Survival after resection of carcinoma of the splenic flexure. Dis Colon Rectum 1991;34:401-3.

6. Nakagoe T, Sawa T, Tsuji T et al. Carcinoma of the splenic flexure: multivariate analysis of predictive factors for clinicopathological characteristics and outcome after surgery. J Gastroenterol 2000;35:528-35.

7. Bae SU, Baek SJ, Hur H, et al. Robotic left colon cancer resection: a dual docking technique that maximizes splenic flexure mobilization. Surg Endosc 2015;29:1303-9.

8. Baran B, Mert Ozupek N, Yerli Tetik N, et al. Difference Between Left-Sided and Right-Sided Colorectal Cancer: A Focused Review of Literature. Gastroenterology Res 2018;11:264-73.

9. Manceau G, Benoist $S$, Panis $Y$, et al. Elective surgery for tumours of the splenic flexure: a French inter-group (AFC, SFCD, FRENCH, GRECCAR) survey. Tech Coloproctol 2020;24:191-8.

10. Ardu M, Bergamini C, Martellucci J, et al. Colonic splenic flexure carcinoma: is laparoscopic segmental resection a safe enough oncological approach? Surg Endosc 2020;34:4436-43.

11. Chenevas-Paule Q, Trilling B, Sage PY, et al. Laparoscopic segmental left colectomy for splenic flexure carcinoma: a single-institution experience. Tech Coloproctol 2020;24:41-8.

12. Fukuoka A, Sasaki T, Tsukikawa S, et al. Evaluating distribution of the left branch of the middle colic artery and the left colic artery by CT angiography and colonography to classify blood supply to the splenic flexure. Asian J Endosc Surg 2017;10:148-53.

13. Martín Arévalo J, Moro-Valdezate D, Garcia-Botello SA, et al. Propensity score analysis of postoperative and oncological outcomes after surgical treatment for splenic flexure colon cancer. Int J Colorectal Dis 2018;33:1201-13.

14. Odermatt M, Siddiqi N, Johns R, et al. Short- and long-term outcomes for patients with splenic flexure tumors treated by left versus extended right colectomy are comparable: a retrospective analysis. Surg Today 2014;44:2045-51.

15. Kim JC, Lee JL, Yoon YS, et al. Robotic left colectomy 
with complete mesocolectomy for splenic flexure and descending colon cancer, compared with a laparoscopic procedure. Int J Med Robot 2018;14:e1918.

16. Wee IJY, Kuo LJ, Ngu JC. A systematic review of the true benefit of robotic surgery: Ergonomics. Int J Med Robot 2020;16:e2113.

17. Catanzarite T, Tan-Kim J, Whitcomb EL, et al.
Ergonomics in Surgery: A Review. Female Pelvic Med Reconstr Surg 2018;24:1-12.

18. Van't Hullenaar CDP, Bos P, Broeders IAMJ. Ergonomic assessment of the first assistant during robot-assisted surgery. J Robot Surg 2019;13:283-8.

(English Language Editor: L. Huleatt)

Cite this article as: Zhang T, Song Z, Zhang Y, Ji X, Jing X, Shi Y, Cheng X, Zhao R. Single-docking robotic-assisted arteryguided segmental splenic flexure colectomy for splenic flexure cancer-a propensity score-matching analysis. J Gastrointest Oncol 2021;12(3):944-952. doi: 10.21037/jgo-21-221 